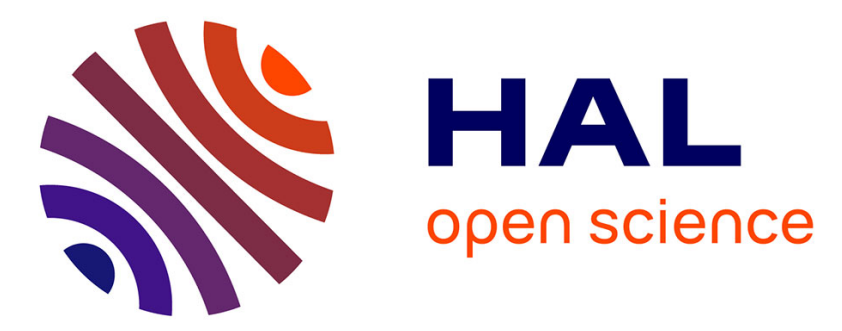

\title{
Image-Based Simulations Show Important Flow Fluctuations in a Normal Left Ventricle: What Could be the Implications?
}

Christophe Chnafa, Simon Mendez, Franck Nicoud

\section{- To cite this version:}

Christophe Chnafa, Simon Mendez, Franck Nicoud. Image-Based Simulations Show Important Flow Fluctuations in a Normal Left Ventricle: What Could be the Implications?. Annals of Biomedical Engineering, 2016, 44 (11), pp.3346-3358. 10.1007/s10439-016-1614-6 . hal-01303067

\section{HAL Id: hal-01303067 \\ https://hal.science/hal-01303067}

Submitted on 15 Apr 2016

HAL is a multi-disciplinary open access archive for the deposit and dissemination of scientific research documents, whether they are published or not. The documents may come from teaching and research institutions in France or abroad, or from public or private research centers.
L'archive ouverte pluridisciplinaire HAL, est destinée au dépôt et à la diffusion de documents scientifiques de niveau recherche, publiés ou non, émanant des établissements d'enseignement et de recherche français ou étrangers, des laboratoires publics ou privés. 


\section{Title page}

Title of the article: Image-based simulations show important flow fluctuations in a normal left ventricle: what could be the implications?

Authors: C. Chnafa ${ }^{1,2}$, S. Mendez ${ }^{1}$ and F. Nicoud ${ }^{1}$

\section{Departments and institutions:}

1. Université de Montpellier - IMAG CNRS UMR 5149 Place Eugene Bataillon. 34095 Montpellier cedex 5 - France.

2. University of Toronto - Mechanical and Industrial Engineering, Biomedical Simulation Laboratory, 5 King's College Road, Toronto, ON M5S 3 G8 - Canada.

Abbreviated title: Flow fluctuations in a normal left ventricle.

\section{For correspondence:}

Christophe Chnafa, University of Toronto - Mechanical and Industrial Engineering, Biomedical Simulation Laboratory, 5 King's College Road, Toronto, ON M5S $3 G 8$ - Canada.

Email: cchnafa@mie.utoronto.ca

Telephone: 416-978-7773

Fax: 416-978-7753

\section{Abstract and key terms}

Abstract: Intra-cardiac flow has been explored for decades but there is still no consensus on whether or not healthy left ventricles (LV) may harbour turbulent-like flow despite its potential physiological and clinical relevance. The purpose of this study is to elucidate if a healthy LV could harbour flow instabilities, using image-based computational fluid dynamics (CFD). 35 cardiac cycles were simulated in a patient-specific left heart model obtained from cardiovascular magnetic resonance (CMR). The model includes the valves, atrium, ventricle, papillary muscles and ascending aorta. We computed phase-averaged flow patterns, fluctuating kinetic energy (FKE) and associated frequency components. The LV harbours disturbed flow during diastole with cycle-to-cycle variations. However, phase-averaged velocity fields much resemble those of CMR measurements and usually reported CFD results. The peak FKE value occurs during the $\mathrm{E}$ wave deceleration and reaches $25 \%$ of the maximum phase-averaged flow kinetic energy. Highest FKE values are predominantly located in the basal region and their frequency content reach more than $200 \mathrm{~Hz}$. This study suggests that high-frequency flow fluctuations in normal LV may be common, implying deficiencies in the hypothesis usually made when computing cardiac flows and highlighting biases when deriving quantities from velocity fields measured with CMR.

Key terms: Left Heart, MRI, Transitional flow, Turbulence, LES, Turbulent Kinetic Energy, Mitral Valve, Atrium, Third Sound, Bruit. 


\section{Introduction}

The hemodynamics of the left ventricle (LV) conveys useful information regarding the heart function ${ }^{25,41}$. Therefore, direct observation of LV flow patterns or indirect measurements of the flow through LV bruits or ejection fraction may signal a normal LV or reflect the presence of an installed pathology. In addition to indicating an abnormal function, there is substantial evidence that the LV hemodynamics can be responsible for the initiation of ventricular remodelling through mechanical stimuli ${ }^{12,36}$. Indeed, LV adaptations involve mechanosensitive feedbacks, which modulate cardiomyocytes architecture and thus cardiac function ${ }^{38}$. Therefore, accurate assessment of the intraventricular flow from smallest to largest scales is of paramount importance to get further comprehension of the role played by the hemodynamics in normal and abnormal LV.

Previous in vivo studies of 4D intra-cardiac flows have been heavily based on filtered velocity data obtained thanks to phase-contrast cardiovascular magnetic resonance $(\mathrm{CMR})^{32}$ imaging. Although comprehensive, CMR velocity mapping is not real-time, but rather measure an averaged heart cycle. Hence, cycle-to-cycle variations and instabilities in the flow cannot be recorded, as the $k$-space is filled over many cardiac cycles ${ }^{15,32}$. Moreover, CMR spatiotemporal resolution precludes the observation of small-scale and fast time-varying flow features in the case of disturbed flow.

Used with caution, image-based computational fluid dynamics (CFD) offers a research tool ${ }^{40}$ able to retrieve all the scales of the instantaneous flow, hence being able to capture highly disturbed flows. However, pioneering CFD studies focused only on the laminar features of the flow $24,29,47,48,52$. While these studies retrieve the large-scale flow features, they were not able to detect fluctuations as such features are likely to be damped by the numerical setup employed ${ }^{50}$. To the best of our knowledge, apart from our previous study ${ }^{10}$, only Le \& Sotiropoulos $^{28}$ have mentioned flow fluctuations in a patient-specific LV model (although without quantifications) and Domenichini et $a .^{13}$ mentioned that in certain conditions LV could be on the edge of "turbulence".

We emphasise that in addition to be controversial, the nature of the flow in normal heart most probably does not comply with the canonical definition of developed turbulence. Due to the flow nature, the Kolmogorov energy cascade does not have time to establish ${ }^{43}$, making the definition of intracardiac turbulence problematic. Nevertheless, non-periodic, 3D fluctuations may appear given the Reynolds number of order 5000, the flow domain complexity and the pulsatile nature of the flow. In the present study, irrespective of the exact nature of the flow, we are referring to cycle-to-cycle flow fluctuations simply as "fluctuations" and we are defining the flow as "disturbed" or "transitional".

Despite its potential importance, little focus has been directed on the disturbed nature of the LV flow. The intrinsic technical limitations of the CMR exams or the numerical strategies generally employed in LV CFD studies are likely to be responsible for the quasi-absence of studies about fluctuations, as they cannot grasp the entire nature of the flow. Fluctuations 
were reported in our previous studies ${ }^{9,10}$ in an abnormal heart and very recently, using a new CMR method to evaluate the intensity and the localization of velocity fluctuations, Zajac et al. ${ }^{53}$ confirmed the presence of non-negligible in vivo level of "turbulent" kinetic energy in abnormal and normal LVs. As the nature of the flow directly affects the characteristic time scales and local levels of flow stresses, we believe that the analysis of the velocity fluctuations can provide a new paradigm in the assessment of the cardiovascular flow and mechanosensitive feedbacks. In addition, characterizing fluctuations might give potential explanation to heart sounds ${ }^{46}$. In this paper we are using image-based simulations ${ }^{10}$ to address the question of whether or not fluctuations are detected in a normal LV. The implications of their existence are then discussed.

\section{Materials and Methods}

In this study, a subject-specific left heart (LH) model and its deformation were extracted from CMR acquisitions. Computational fluid dynamics (more precisely large-eddy simulations) was used to study the flow in this model.

\subsection{Patient-specific domain}

CMR images were obtained from a healthy subject of 26 years old. The 4D image set consists in 20 three-dimensional images of spatial resolution $5.0 \times 1.1 \times 1.1 \mathrm{~mm}^{3}$, which correspond to $21 \times 256 \times 256$ voxels. The subject cardiac cycles lasted on average $T=750 \mathrm{~ms}$. We selected one 3D image (referred to as the native image) at an arbitrary moment of the heart cycle and we imported the corresponding volumetric data into an image processing software (ScanIP; Simpleware Ltd., Exeter, UK). We segmented a 3D domain covering all the space occupied by blood in the LH cavities using a thresholding method and a smoothing procedure ${ }^{42}$. Figure SO1 in the supplementary materials shows a long axis slice of the CMR exam and the segmentation resulting from the thresholding method. The 3D domain includes the ventricle, atrium, four pulmonary veins, the ascending aorta and the papillary muscles. We chose to simulate the entire $L H$, even if the focus is on the LV, in order to mitigate the uncertainty related to the swirled and skewed flow at the mitral valve $(\mathrm{MV})^{10,34}$. Figure 1 shows the domain used for the CFD. The extracted geometry was imported in a mesh generator (Gambit, ANSYS) to generate the grid of the native numerical domain.

A set of numerical treatments was applied to the CMR images and the native grid in order to generate a time-varying computational domain representing the physiological deformations of the LV. The resulting LV volume (as defined in Fig. 1 i.e. the LV volume is delimited by the $\mathrm{AO}$ and MV annulus) varies from $109 \mathrm{~mL}$ (end diastolic volume) to $41 \mathrm{~mL}$ (end systolic volume). The stroke volume is $68 \mathrm{~mL}$ and the ejection fraction is $62 \%$, which falls within the normal physiological range ${ }^{31}$. Details are provided in previous studies ${ }^{9,10}$. 

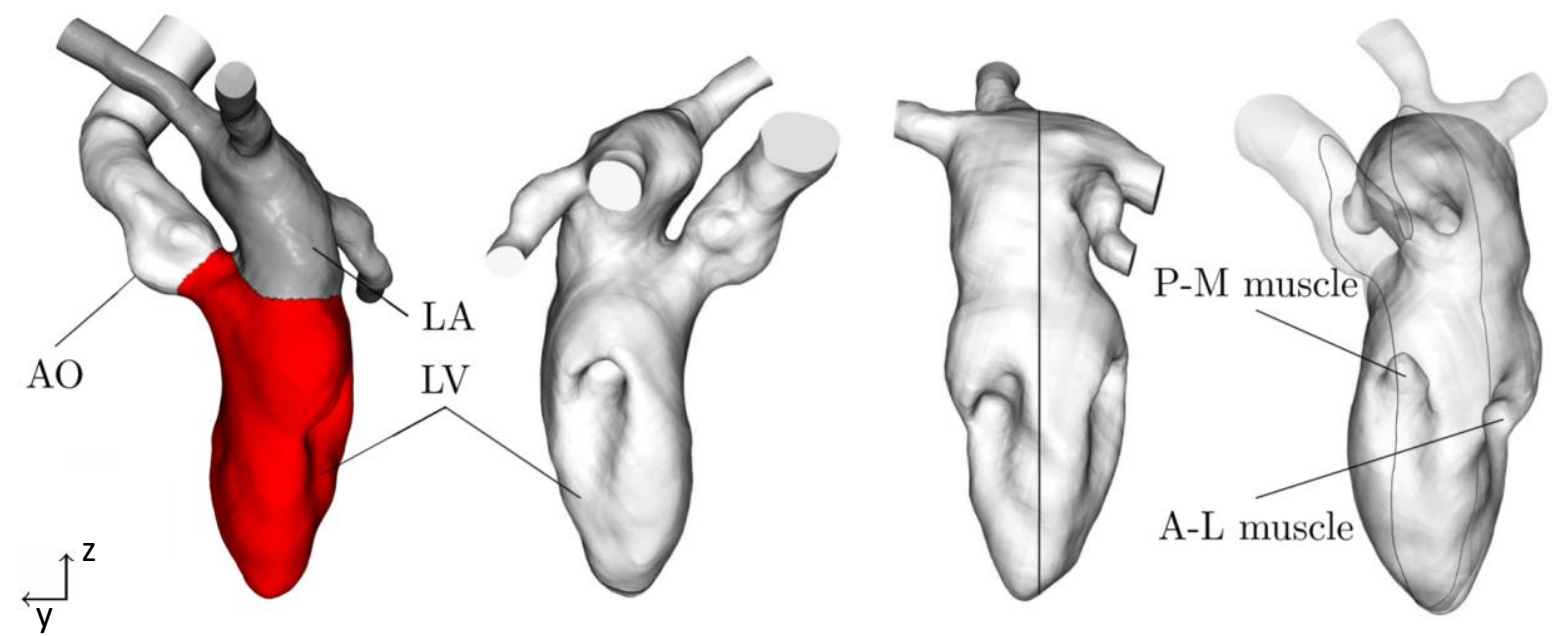

Figure 1 - Full human LH extracted from CMR images. The same domain is shown from four different points of view. The inlets and outlet flow extensions are visible in the left figure. The left ventricle (LV), left atrium (LA), Aorta (AO), Anterio Lateral (A-L) and Postero Medial (P-M) papillary muscles are indicated. A black line passing through the $\mathrm{LH}$ indicates the position of the slice which will be used to display the velocity field in the next section. The red volume is the volume used for all the volumetric integrations over the LV. The volume is delimited by the aortic and mitral annulus.

The aortic valve (AV) and the MV are difficult to extract from the medical images as they are thin and highly moving structures. However, as valves have an impact on the flow structure ${ }^{5}$, low-order models were included in the domain. The valve models have been described in detail in a previous work ${ }^{9,10}$ and are only briefly recalled here. Annuluses are reconstructed by inspecting the medical images and placing markers manually on the native 3D domain. The motion of these markers is then obtained thanks to the heart motion extracted from the medical images. We modelled the AV, which has a moderate impact on the ventricular flow, as a planar region at the $\mathrm{AV}$ annulus being alternatively permeable and impermeable depending on the phase in the cardiac cycle. We modelled the MV using parameters measured in the CMR exam: the leaflet length, annulus position and MV open area. Figure S02 in the supplementary materials depicts the measurements of the mitral open area and the measurements along the diastole. The median opened area is $A_{M V}=5.18 \mathrm{~cm}^{2}$, which falls in the normal physiological range ${ }^{31}$. The open area available for the fluid flow is modelled as an ellipse of axis $a=15 \mathrm{~mm}$ and $b=11 \mathrm{~mm}$. Knowing the MV leaflets position during the heart cycle, their effect on the blood flow is accounted for by using an immersed boundary method ${ }^{9}$. For this purpose, the leaflets representations are given a thickness so that a few mesh nodes are located within the valves. Then, the fluid velocity is imposed to zero within the leaflets.

\subsection{Fluid boundary conditions}

A no-slip condition was applied to the moving heart walls. The inflow boundary conditions at the pulmonary veins were computed by assuming that the mitral and aortic valves are either closed-open or open-closed at each instant. Under this assumption, the flow rate entering the flow domain is related to the time evolution of either the atrium volume or the atrioventricular volume, by using the mass conservation principle. In other words, the boundary 
conditions are derived from the medical images ${ }^{9}$ and the associated heart deformations over time. The resulting waveform is applied as a boundary condition at the four pulmonary veins, assuming an equipartition between the four inlets. The same inlet waveform and wall deformation are applied for each cycle, precluding the generation of cycle-to-cycle variations into the flow through boundary condition. A traction free outflow condition is applied at the outlet. Figure 2 displays the resulting flow rates at the AV and the MV.

Systole

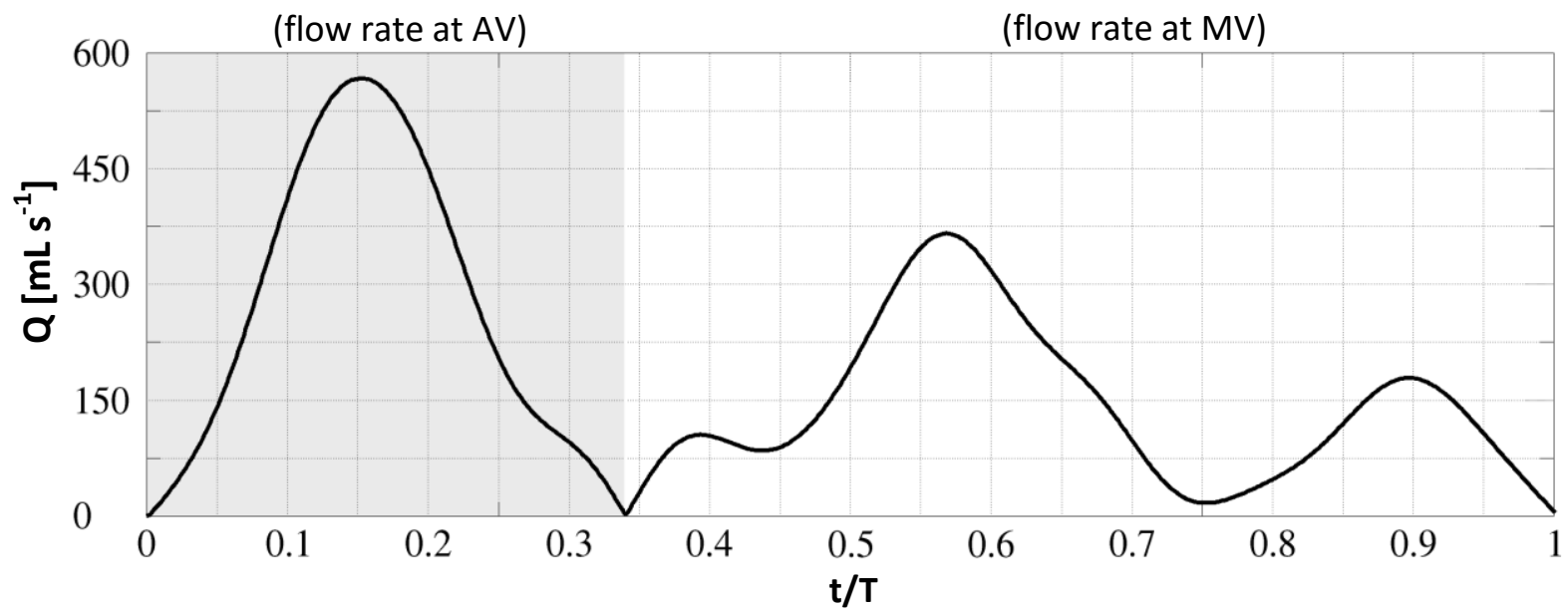

Figure 2 - Flow rates at the aortic valve (null during diastole) and at the mitral valve (null during systole). The grey area delimits the systolic phase from 0 to $0.34 \mathrm{~T}$. Note that a normal time ratio of one-third (systole) to two-thirds (diastole) is respected.

The $E$ wave peak $\left(0.57 \mathrm{~T}\right.$ ) corresponds to an entering flow rate of $365 \mathrm{~mL} \mathrm{~s}^{-1}$ in the ventricle. The A wave peak occurs at $0.89 \mathrm{~T}$ and corresponds to an entering flow rate of $180 \mathrm{~mL} \mathrm{~s}$ resulting in a E/A ratio of 2 . The maximum Reynolds is $R e=4524$ at the $M V$ tips using the area $A_{M V}$, the effective mitral mean diameter $D=2 \sqrt{A_{M V} / \pi}$, the maximum flow rate and a constant kinematic viscosity $v=4.0 \times 10^{-6} \mathrm{~m}^{2} \mathrm{~s}^{-1}$. The Stokes number is $\beta=D^{2} / \mathrm{v} T=220$ and the Strouhal number is $S t=\beta / \operatorname{Re}=0.049$. Table 1 summarizes the main characteristics of the simulation compared to normal values. 


\begin{tabular}{|c|c|c|}
\hline & Present simulation & Normal range \\
\hline Heart rate $\left(\right.$ Beat $\mathrm{min}^{-1}$ ) & 85 & $60-100$ \\
\hline E/A ratio & 2.0 & $1.0-2.0$ \\
\hline EDV (mL) & 109 & $65-240$ \\
\hline ESV (mL) & 41 & $16-143$ \\
\hline $\mathrm{SV}(\mathrm{mL})$ & 68 & $55-100$ \\
\hline Cardiac output $\left(\mathrm{L} \mathrm{min}^{-1}\right)$ & 5.8 & $4.0-8.0$ \\
\hline Ejection fraction (\%) & 62 & $55-70$ \\
\hline MV Open area $\left(\mathrm{cm}^{2}\right)$ & 5.18 & $4.0-6.0$ \\
\hline $\mathrm{U}_{\max }$ at $\mathrm{MV}\left(\mathrm{m} \mathrm{s}^{-1}\right)$ & 0.7 & $0.6-1.0$ \\
\hline $\mathbf{R e}_{\max }$ at $\mathbf{M V}$ & 4524 & $4146-5642$ \\
\hline
\end{tabular}

Table 1 - Main flow characteristics of the LV simulated compared to LV normal ranges. Note: the range of the Reynolds number is computed from $U_{\max }$, using the normal range of the MV open area, and the viscosity used in the simulation $\left(v=4.0 \times 10^{-6} \mathrm{~m}^{2} \mathrm{~s}^{-1}\right)$. EDV: end-diastolic volume; ESV: end-systolic volume; SV: stroke volume.

\subsection{Numerical setup}

The Navier-Stokes equations (NS) are solved using large-eddy simulations and the finitevolume method as implemented in the YALES2BIO solver ${ }^{10,33}$ (www.math.univmontp2.fr/ yales2bio). The YALES2 solver and its biomechanical spin-off YALES2BIO have been extensively validated in prior studies. Simple and idealized geometries have been used to validate the solver and can be found here ${ }^{8}$. The code has shown its capability to reproduce accurately industrial configuration against another CFD code and against experimental measurements via Particle Image Velocimetry (PIV) ${ }^{1}$. Moreover, Toda et al. ${ }^{2}$ studied the accuracy of the LES model we are using in this study thanks to an experimental setup of a pulsatile jet impinging a flat-plate in the presence of a cross-flow. This configuration shows a strong similarity with the mechanisms present in the heart and reported in the present study. Toda et al. $^{2}$ showed the capability of the LES model in reproducing accurately this experimental configuration against PIV.

The code uses spatial gradients computed with a centred fourth-order scheme. The timeadvancement scheme of the NS equation is an explicit low-dissipative, low-storage, four-step Runge-Kutta scheme recast in an arbitrary Lagrangian-Eulerian formalism. The pressure term is treated with the Chorin's projection-correction method. We modelled the subgrid-scales with the Sigma eddy-viscosity model ${ }^{35}$. We discretized the LH geometry using ten million tetrahedral elements. The average edge length of the tetrahedra was close to $0.55 \mathrm{~mm}$ during diastole. See Fig $\mathrm{SO} 3$ in the supplementary materials for a representation of the computational mesh. The time step calculation was based on a Courant-Friedrichs-Lewy stability number of 0.9 , which resulted in a temporal resolution varying from $0.2 \mathrm{~ms}$ during the beginning of diastole to $0.5 \mathrm{~ms}$ during diastasis. Five cycles were simulated to wash out the initial conditions and statistics were accumulated over 30 additional cycles.

As a common practice when using properly resolved LES, all quantities are computed from the resolved velocity field in this study ${ }^{4,43}$. We note that during our computations the sub- 
grid scale viscosity remains low in the LV during the whole the heart cycle, showing that the sub-grid scale model dissipates a moderate amount of energy. Figure S04 (supplementary materials) shows the ratio between the sub-grid scale viscosity and the fluid viscosity. As expected, the sub-grid scale viscosity is virtually zero during the systole and has moderate values, but non-null, during the most turbulent part of the cycle. The maximum mean ratio is approximatively 0.2 during the more turbulent part of the cycle. To go further, an estimation of the Pope criterion ${ }^{44}$ can be computed as $k_{\text {sgs }} / k \approx 3 C \Delta k / 2 \pi L^{43}$ where $\mathrm{C}=1.5, \Delta=0.55$ $\mathrm{mm}$ and, $\mathrm{L}=0.04 \mathrm{~m}$ being the characteristic length of the largest structures. For the present LES, $k_{\text {sgs }} / k=6 \%$ which remains three times below the $15-20 \%$ threshold usually used to evaluate if a LES is sufficiently resolved ${ }^{44}$.

\section{Results}

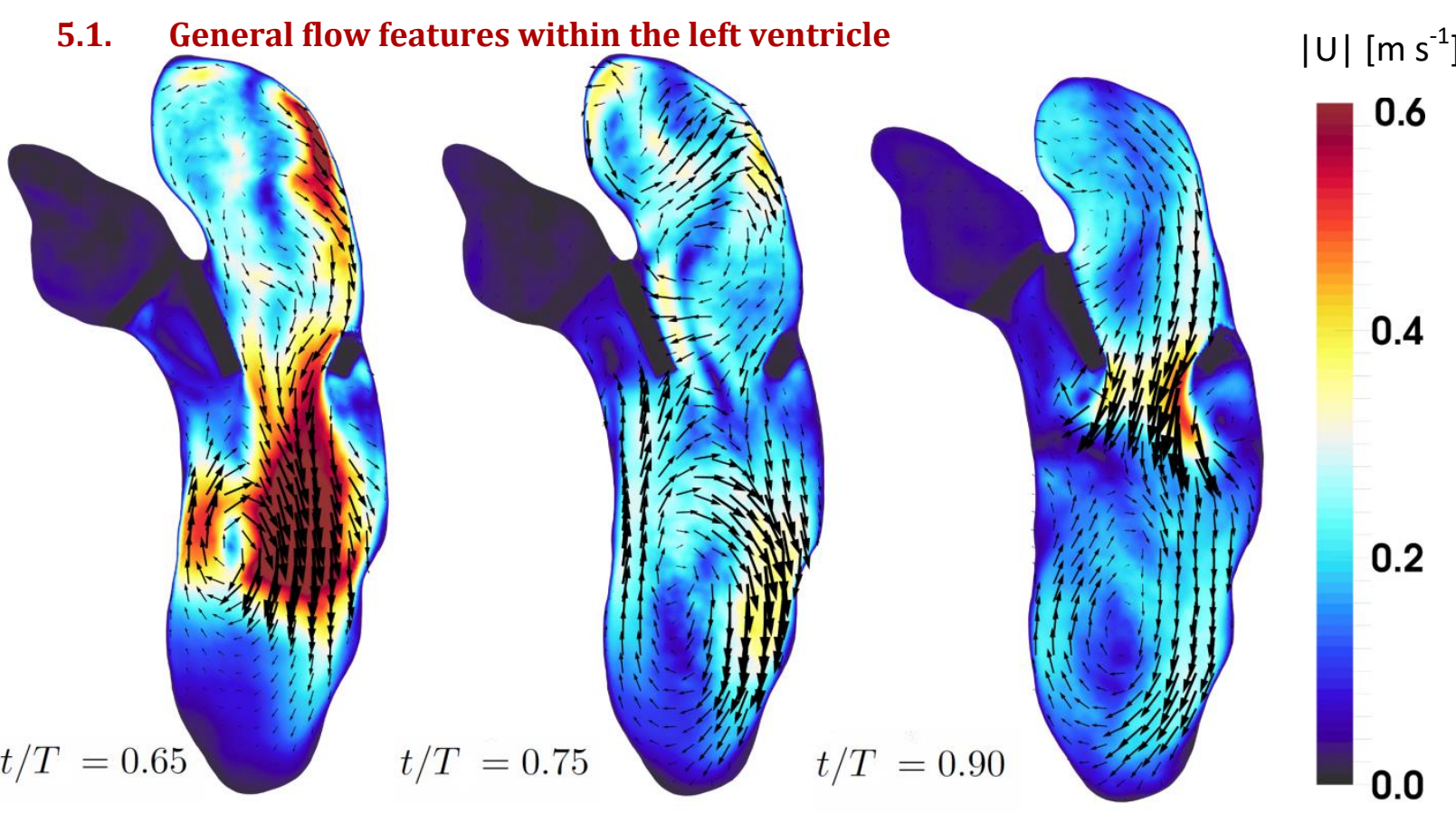

Figure 3 - Phase-averaged diastolic velocity field over a cutting plane through the LH (see Fig. 1 for the plane position). The MV and the AV are depicted in black. Colour map represents the velocity magnitude. The velocity vectors are plotted each $3.7 \mathrm{~mm}$. The figure shows the classically reported structure of the LV diastole: the $E$ wave vortex ring during the second half of the $E$ wave filling $(0.65 \mathrm{~T})$, the recirculating cell during the diastasis $(0.75 \mathrm{~T})$ and the $A$ wave filling (0.9 T).

Figure 3 displays phase-averaged flow velocity solutions projected onto the slice indicated in Fig. 1. Atrium and aorta are displayed, but the description focuses on the LV flow. As flow patterns in ventricles are dominated by the diastolic inflow, we only show salient moments of the diastolic phase.

During the E wave filling, a jet enters the ventricle and generates a vortex ring, quickly regionally constrained due to its interaction with the LV surface. The vortex ring becomes 
asymmetric: the unconstrained part of the vortex moves and grows as shown by the velocity field plotted at $0.65 \mathrm{~T}$. Therefore, at the end of the $\mathrm{E}$ wave, the initial vortex ring forms a recirculating cell, as shown by the velocity field plotted at $0.75 \mathrm{~T}$, which takes up the entire ventricle for the rest of the cycle. After diastasis, at 0.9 T, a new jet enters the ventricle as displayed in the right figure. This second injection of fluid corresponds to the A wave. This vortex ring is less intense than the $E$ wave vortex and remains more coherent, as it does not impinge with the heart wall. At the end of diastole, the MV closes and the AV opens. The systolic phase begins; the ventricle volume decreases and blood is ejected from the LV. Overall, the simulation retrieves the classically reported large-scale flow structures ${ }^{26,32,51}$.

In addition, small-scale structures are present in the LV, but they are not visible on the phase-averaged fields. Figure 4 displays instantaneous velocity solutions at $0.75 \mathrm{~T}$ (left and middle plots) for two consecutive heart cycles while the right plot shows the phase-averaged flow for the same time (also displayed in Fig. 3). Instantaneous velocity solutions exhibit cycle-to-cycle fluctuations, looking like random flow variations in time and space, mainly from the impingement of the $E$ wave vortex ring (at roughly $0.65 \mathrm{~T}$ ) to the end of diastole. Cycleto-cycle differences are visible in the entire LH while the phase-averaged flow (right plot) shows the large-scale features usually reported in in vitro, in silico and in vivo studies.

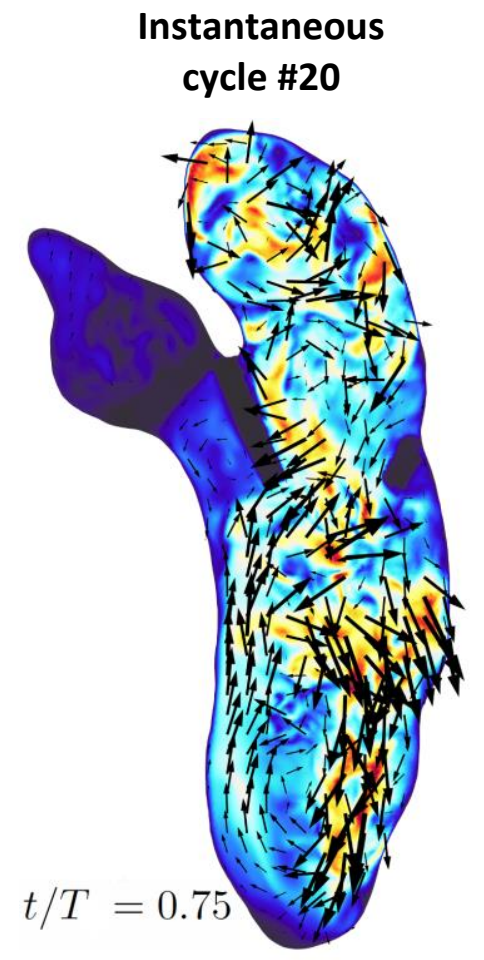

\section{Instantaneous cycle \#21}

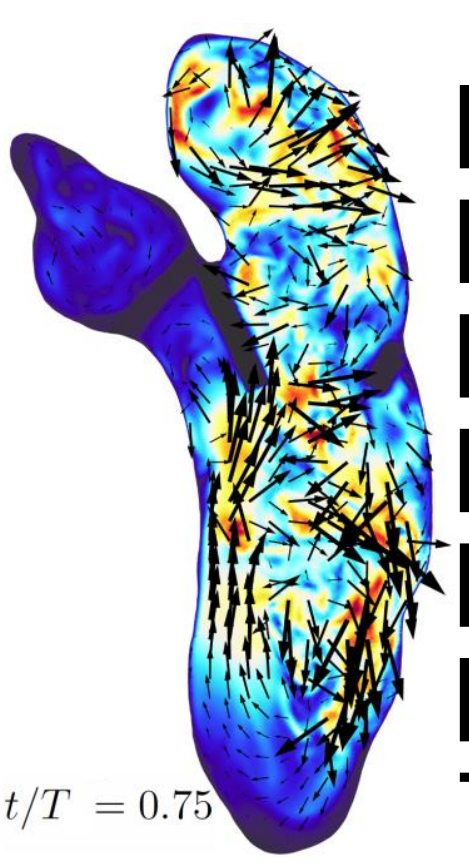

\section{Phase-averaged over 30 cycles}

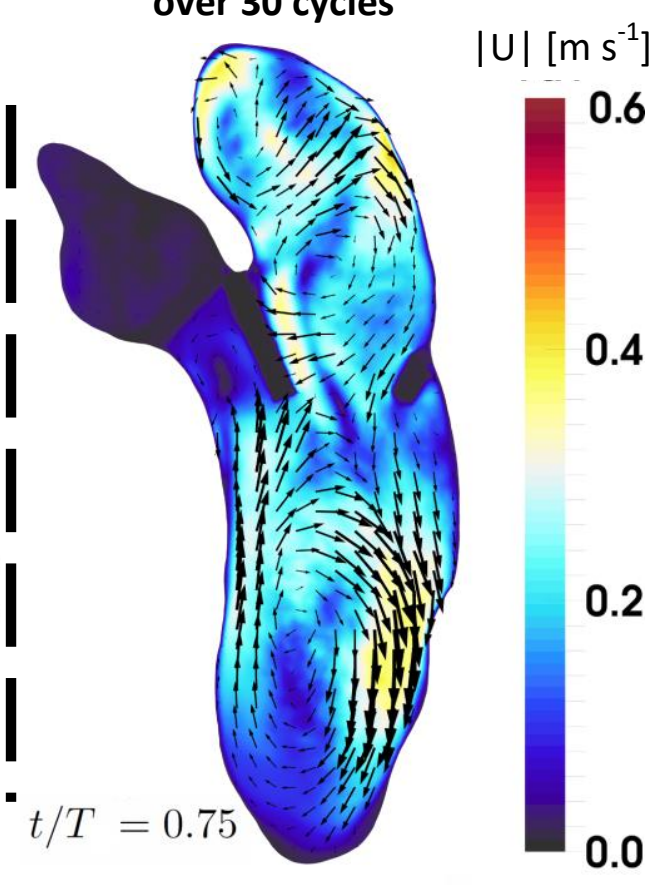

Figure 4 - Left and middle plots: instantaneous velocity fields at $0.75 \mathrm{~T}$ for two consecutive heart cycles. Right plot: phase-averaged velocity field over thirty heart cycles at $0.75 \mathrm{~T}$. Note the large cycle-to-cycle fluctuations while the phase-averaged flow shows the classically reported flow pattern. 


\subsection{Regional repartitions of the fluctuations in the LV}

In order to study the regional distribution and quantify these fluctuations we computed the difference between the phase-averaged velocity components $U_{i}$ and the instantaneous velocity components $u_{i}(\mathrm{i}=1,2,3)$. The fluctuating part of the fluid velocity is,

$$
u_{i}^{\prime}=U_{i}-u_{i}
$$

and we defined the fluctuating kinetic energy (FKE) per unit volume as,

$$
F K E=\frac{\rho}{2}<u_{i}^{\prime} u_{i}^{\prime}>\text {, }
$$

where $<.>$ denotes phase-averaged values, and with implicit sum. Figure 5 displays the FKE in the LV when the vortex ring interaction with the heart wall occurs (left figure), during diastasis (centre figure) and at the A wave peak (right figure).

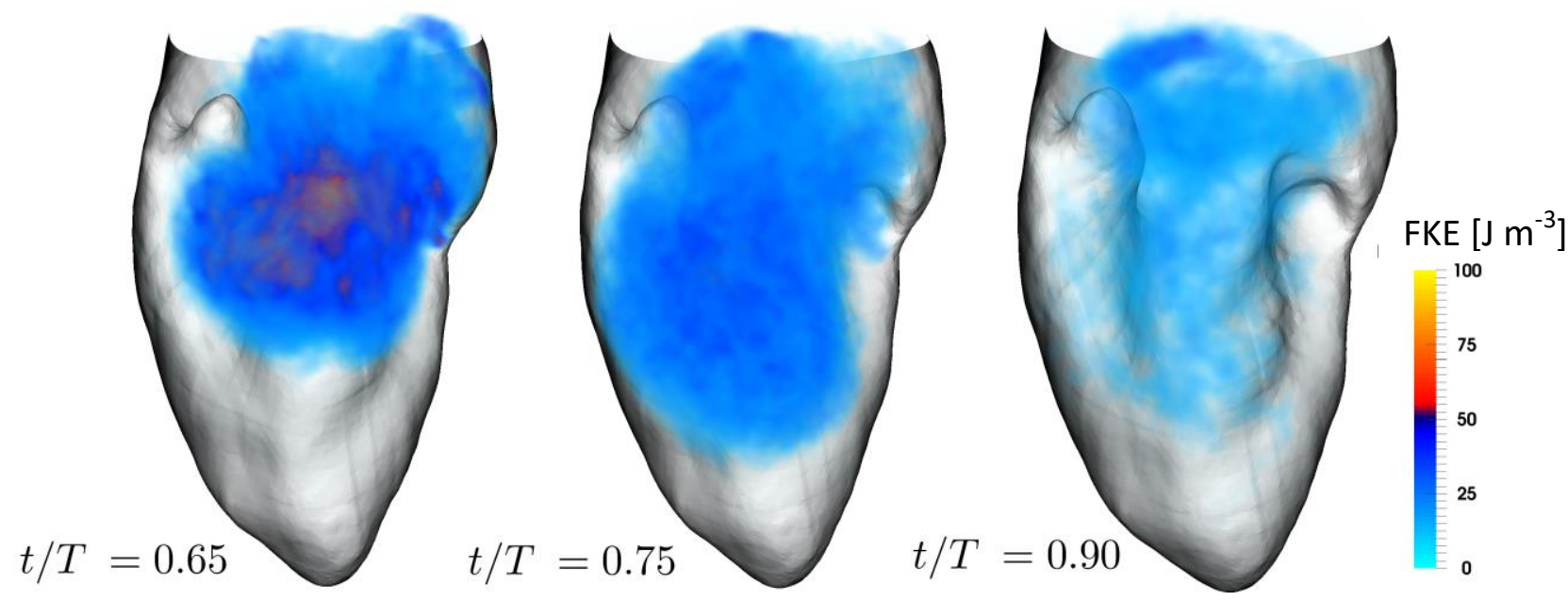

Figure 5 - 3D volume rendering of the FKE in the LV during the second half of the $E$ wave peak (left, $0.65 \mathrm{~T}$ ), diastasis (middle, $0.75 \mathrm{~T}$ ) and at the A wave peak (right, $0.9 \mathrm{~T}$ ). The opacity is set to $0 \%$ for FKE values below $10 \mathrm{~J} \mathrm{~m}^{-3}$ and then grows linearly from $0 \%$ to $100 \%$ for the maximum FKE values displayed.

We also provide an animation of the volumetric rendering of the FKE in the LV during the heart cycle, as supplementary online material. During the heart cycle, the FKE ranges from zero to $150 \mathrm{~J} \mathrm{~m}^{-3}$ in the LV. Fluctuations around $60 \mathrm{~J} \mathrm{~m}^{-3}$ are observed in the vortex ring and its wake. The highest FKE values are predominantly located in the basal region, in the upper third of the LV, when the vortex ring begins to interact with the heart wall at $0.62 \mathrm{~T}$. After this interaction, fluctuations are visible in the major part of the LV, while their intensity decreases throughout the rest of the cycle. Values under $10 \mathrm{~J} \mathrm{~m}^{-3}$ are mainly detected in the LV apical area. Later during the cycle, fluctuations are generated in the wake of the A wave vortex ring and remain in the basal region. Fluctuations are dissipated during systole.

\subsection{Time-frequency analysis of the fluctuations}

In order to describe the energetic distribution of the fluctuating part of the velocity $u_{i}{ }^{\prime}$ over the different flow frequencies, we computed spectrograms for different locations in the LV. 
Spectrograms have been preferred to the energy-frequencies study since the flow is here non-ergodic and highly transient, which precludes the classic use of such approach. We decomposed each velocity signal in 800 windows, applied a Hann window with 50 \% overlapping and used a short-time Fourier transformation. The resulting spectrograms are then phase-averaged over the 30 cycles. Spectrograms computed with different overlapping and window functions displayed only small differences, which guarantee that the following results are robust to the details of the signal processing. Figure 6 displays the computed spectrograms at three Eulerian locations for the fluctuating energy.

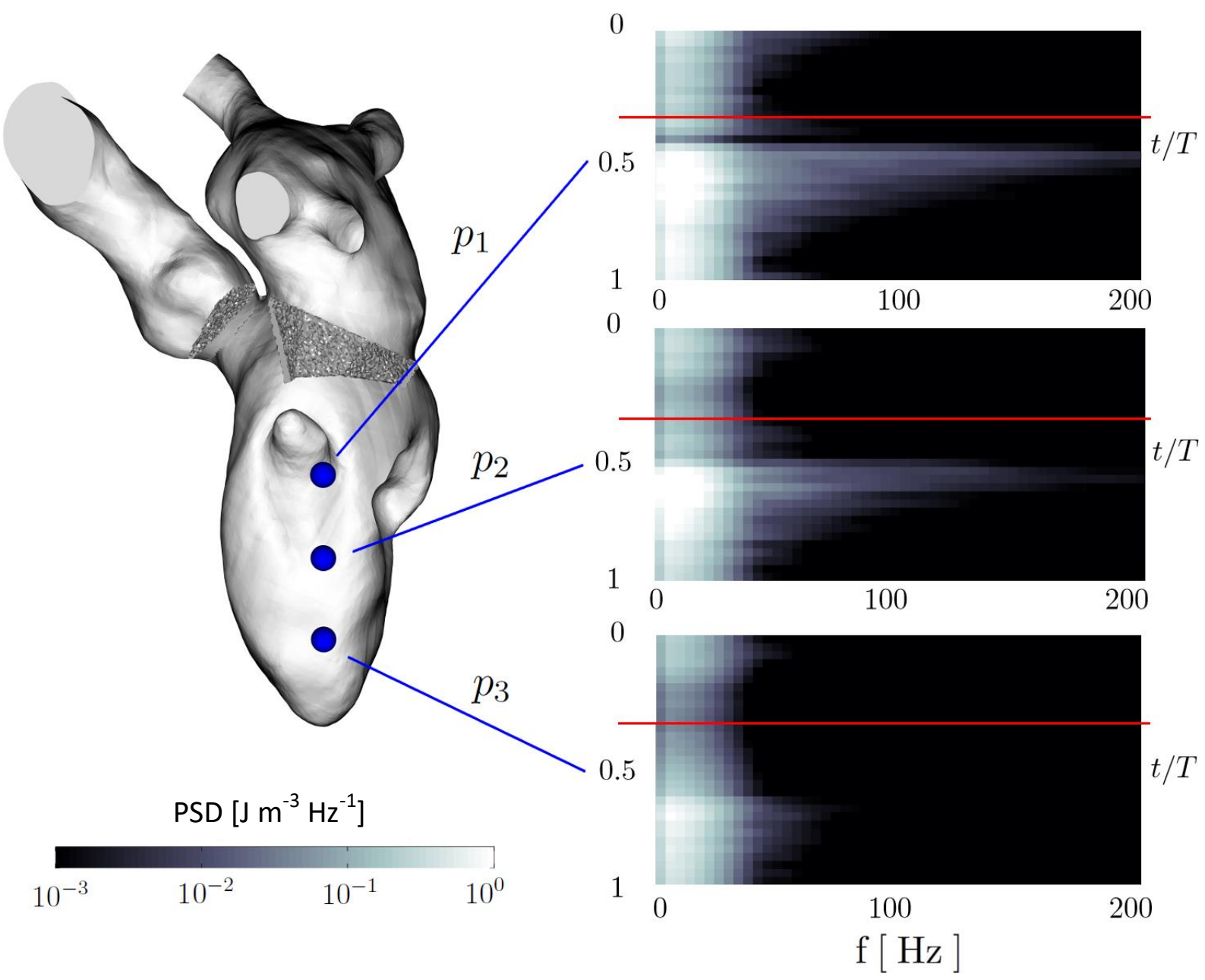

Figure 6 - Time-frequency representation of the Power Spectral Density (PSD) in log scale of the velocity fluctuations $u^{\prime}$ at three locations. Each horizontal slice of a spectrogram exhibits the frequency spectrum of the flow at a specific time. Red lines on spectra delimit systole from diastole. The $\mathrm{LH}$ is made transparent. Atrium, open mitral valve and closed aortic valve are displayed. The three Eulerian probes $p_{1}, p_{2}$ and $p_{3}$ are located as shown in the left hand side view (blue spheres).

The diastolic flow features broad ranges of frequencies at probes $p_{1}$ and $p_{2}$ while $p_{3}$ location displays lower frequencies. During the first half of diastole, the energy in the LV rises and is transferred from lower to higher frequencies. At location $p_{1}$, the broader range of frequencies occurs at roughly $0.65 \mathrm{~T}$, while the maximum is reached at $0.77 \mathrm{~T}$ for $p_{2}$ and $0.9 \mathrm{~T}$ for $p_{3}$. The frequency content of the fluctuations is in a range of frequencies up to $200 \mathrm{~Hz}$ for $p_{1}, 180$ 
$\mathrm{Hz}$ for $p_{2}$ and $70 \mathrm{~Hz}$ for $p_{3}$. For the three locations $p_{1}, p_{2}, p_{3}$, beside the short time windows when higher frequencies were visible, more than $95 \%$ of the energy remains concentrated in frequencies lower than $35 \mathrm{~Hz}$.

\subsection{Integrated fluctuating kinetic energy}

Figure 7 displays the FKE and the averaged velocity kinetic energy, integrated over $V(t)$, the time varying volume of the $L V$. The volume $V(t)$ is delimited by the $A O$ and $M V$ valve annulus as shown in Fig. 1. We defined the integrated FKE as,

$$
E_{k}(t)=\frac{\rho}{2} \int_{V(t)}<u_{i}^{\prime} u_{i}^{\prime}>d V
$$

And the integrated average flow kinetic energy as,

$$
E(t)=\frac{\rho}{2} \int_{V(t)}<U_{i} U_{i}>d V .
$$

$E_{k}$ ranges from 0.1 to $1.5 \mathrm{~mJ}$. During diastole, the increase of $E_{k}$ measured throughout the LV chamber is associated with the beginning of the $E$ wave. The FKE energy reaches its peak value during the inflow deceleration at $0.71 \mathrm{~T}$, occurring $0.12 \mathrm{~T}$ after the $\mathrm{E}$ wave peak. The $\mathrm{E}$ wave peak energy of the phase-averaged flow has an energy of $5.5 \mathrm{~mJ}$, which falls into the range of measurements reported with $\mathrm{CMR}^{3,23}$. The amount of FKE decreases during the rest of the diastole with a slight inflexion occurring during the last third of the A wave. The relaminarization continues during systole while a peak of averaged velocity energy occurs because of the flow ejection through the aortic valve. Note that this peak seems 3 to 4 times higher than the values reported with $\mathrm{CMR}^{3,23}$. We stress that in contrast to $E_{k}$ or, the diastolic peak of $E$, the peak value of $E$ during systole is sensitive to the choice of volume of integration. As a sensitivity study, we integrated the energies with different volumes to show that the systolic peak show the same values as reported with $\mathrm{CMR}^{3,23}$ when the aortic root is not considered in the integration. See Fig. S05 of the supplementary materials.

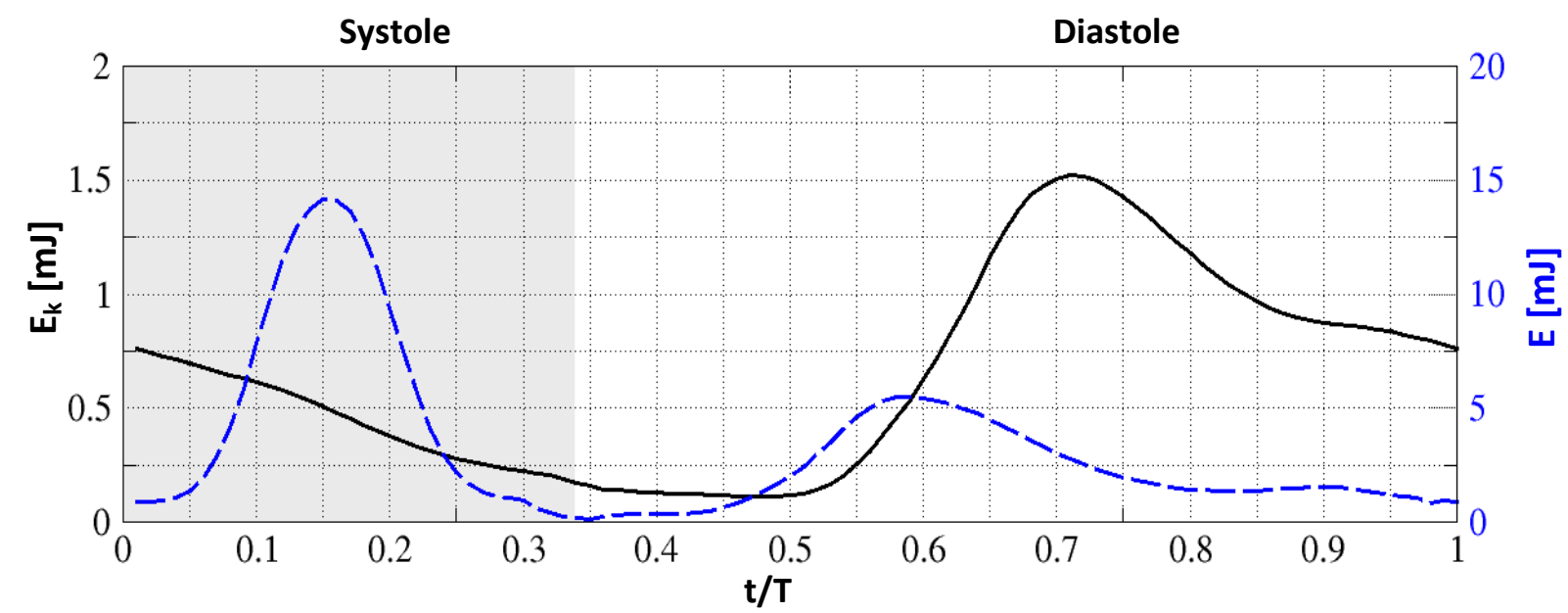

Figure 7 - Fluctuating (black solid line) and averaged (blue dashed line) velocity kinetic energy integrated over the LV during a heart cycle. 


\subsection{Impact of the fluctuations on the wall shear stress}

In order to demonstrate the consequence of these fluctuations on a hemodynamic factor, we computed the wall shear stress (WSS) in the LV for two consecutive cycles and for the phase-averaged velocity field. This estimation is a first approximation since the surface of the ventricle is simplified. The WSS was computed as the multiplication of the total fluid stress and the surface traction vector ${ }^{6}$. Figure 8 shows the resulting patterns over a part of the LV inner surface during diastasis. Cycle-to-cycle differences are important in term of patterns and local intensity while the phase-averaged pattern is more evenly distributed. The magnitude of the phase-averaged WSS falls into previously reported range in the LV ${ }^{37}$.
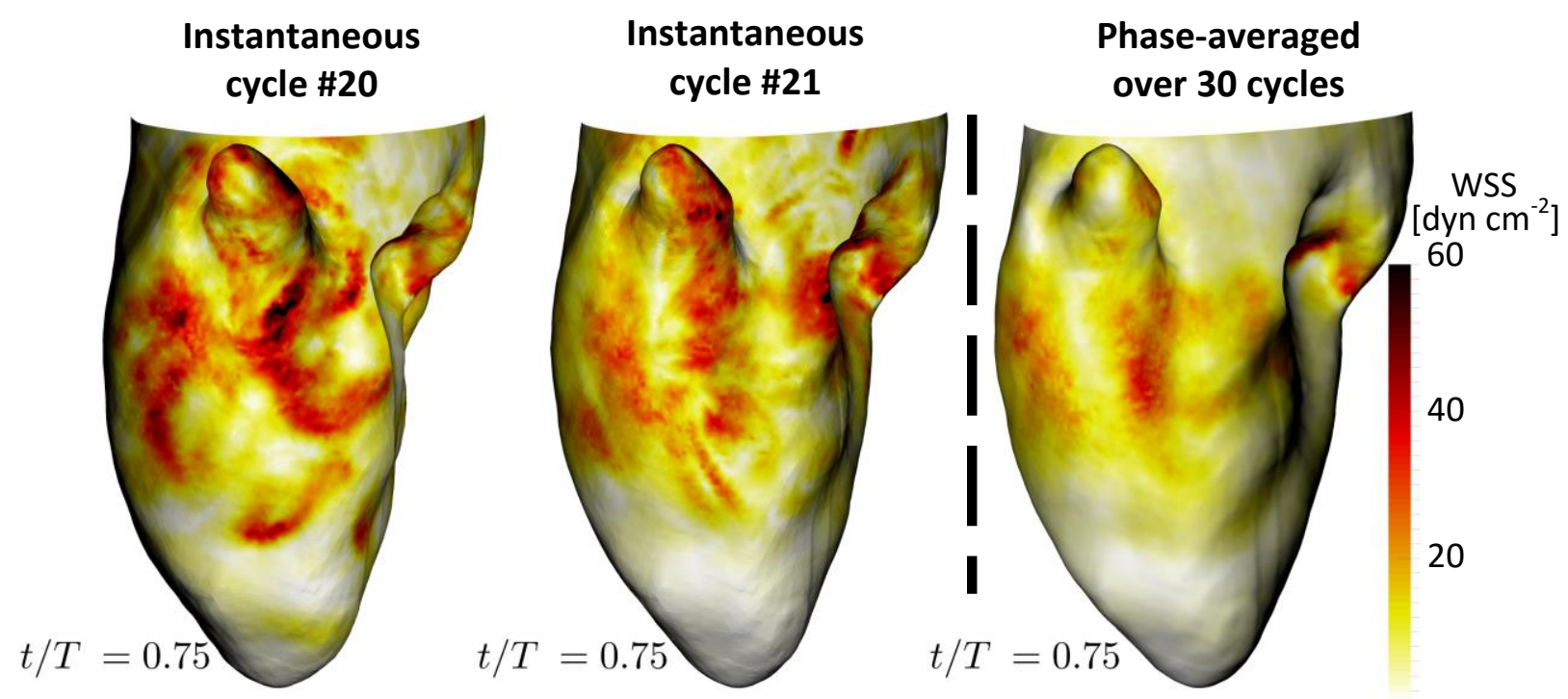

Figure 8 - Left and middle plots: instantaneous wall shear stress maps at $0.75 \mathrm{~T}$ for two consecutive heart cycles. Right plot: phase-averaged wall shear stress map over 30 heart cycles at $0.75 \mathrm{~T}$. Note the large cycle-to-cycle differences.

\section{Discussion}

\subsection{Summary}

The LV large-scale flow features we described i.e., the blood ejection, the two vortex rings for the $E$ and $A$ waves and the recirculating cell, are in accordance with the numerous observations performed in vivo ${ }^{26,32}$, in silico ${ }^{13,34}$ and in vitro ${ }^{19,45}$. However, our computation reveals also large velocity fluctuations, which are usually not reported in silico but which are in line with the results of previous experimental work that used both simplified ventricle geometries and inflow boundary condition ${ }^{14,45}$. The presence of these fluctuations is due to the conjunction of the complex geometry of the LV, the high Reynolds number of the E wave jet and several normal and known mechanisms of the diastolic flow:

O the shear layer instabilities of the Kelvin-Helmholtz type generated by the jets during the $E$ and $A$ wave,

O the interaction between the $E$ wave vortex ring and the wall of the ventricle,

o the flow decelerations associated with an adverse pressure gradient after each filling wave, which favours flow instabilities. 
Consequently, the velocity fluctuations are observed during diastole, primarily when the $E$ wave jet impacts the heart wall and during the subsequent flow deceleration. During systole, the fluid undergoes an acceleration that dampens flow instabilities. The overall quantification of the fluctuations indicates a clear scenario: the flow presents several regimes from laminar, to transitional if not turbulent, at each heartbeat. The fluctuations during the transient phase are not negligible: during diastole, the peak ventricle-averaged FKE value occurring during the $\mathrm{E}$ wave deceleration reaches $25 \%$ of the maximum phase-averaged flow kinetic energy.

The fluctuations are not restrained to a specific location and are observed in the whole ventricle. However, their intensity and range of frequency vary in space. They exhibit low intensity and limited frequency range in the apex area while they show high intensity and broader range of frequencies in the first third of the LV during $30 \%$ of the diastole. Should it be recalled, these fluctuations are just and only due to the non-linearity of the flow equations since the computational domain and boundary conditions are fully periodic in this study.

\subsection{Relationship with previous in silico studies}

Despite never being studied, direct or indirect evidence of flow instabilities in LV has been previously reported. Today, the research tool most capable of detecting and characterizing instabilities in the heart is computational fluid dynamics. However, to the best of our knowledge, the only patient-specific studies evoking fluctuations are ours in a heart with restrictive filling ${ }^{9,10}$ and a study by Le $\&$ Sotiropoulos ${ }^{28}$ who mentioned "transitions to a weak turbulent state" after the impingement of the E wave vortex ring, without further analysis. In an idealized geometry, Domenichini et $a l .{ }^{13}$ mentioned that for a Strouhal number of St = 0.05 and a Stokes number of $\beta=81$ the flow undergoes a transition to a weak turbulent regime in their simulation. They added that a decrease of the Strouhal number, especially with large Stokes number, could trigger a transition to a turbulent regime. Our results confirm their statement, considering the present patient-specific model corresponds to a Strouhal number of $\mathrm{St}=0.0486$ and a Stokes number of $\beta=220$ during the $E$ wave.

The scarcity of reports of fluctuations using CFD is actually not surprising. The majority of the pioneering LV CFD used low resolutions in space and/or dissipative numerical schemes, which can spuriously eliminate flow fluctuations. We stress that in addition to using fourthorder, non-dissipative schemes, the number of elements used for the present simulation is comparable to recent studies using direct numerical simulations $s^{34,51}$ and two to three orders of magnitude larger than the other pioneering studies available $24,29,47,48,52$. These observations call into question the underlying idea of laminar flow in the LV. Indeed, we think that the main reason why reports of flow instabilities are rare in the LV CFD literature is the widely held conception that healthy LV flows are laminar. Undeniably, irrespective of flow phenotype, main large-scale hemodynamic features, namely jets, recirculating cell, and ejection can be retrieved. However, transient or turbulent state features small-scales phenomena that cannot be retrieved under the laminar hypothesis. Therefore, as shown recently in aneurysms CFD by Valen-Sendstad \& Steinman ${ }^{50}$, our results suggest that the laminar assump- 
tion often made implicitly, should be reconsidered in LV CFD if one wants to simulate all the flow features.

\subsection{Relationship with previous in vivo measurements}

It is worth noting that any CFD study where flow instabilities are not properly represented cannot address the prevalence of ventricular bruits and murmurs ${ }^{31,46}$. Among the sounds usually reported, the $S_{3}$ bruit is a low-frequency brief sound harbouring a main frequency content in the range of $10-100 \mathrm{~Hz}^{22}$ and occurring at the end of the $E$ wave, $120-200 \mathrm{~ms}$ after the start of the diastole ${ }^{11,22}$. Although the genesis of this sound is controversial, it is widely reported that "vibrations" occurring during the deceleration of the $E$ wave generate this sound ${ }^{20,27}$. These "vibrations" may be generated by the interaction of the flow instabilities with the LV tissue.

The fluctuations measured in the present study match the characteristics of the third sound $S_{3}$. While we do not directly prove that the reported fluctuations are responsible for the $S_{3}$ bruit, extrapolation from our results would support a cause-and-effect relationship ${ }^{46}$. Indeed, the present time-frequency analysis showed consistent results regarding a) the $S_{3}$ main frequency content previously reported ${ }^{22}(10-100 \mathrm{~Hz})$ as well as b) the time window when the most intense fluctuations occurred ${ }^{11,22}$ (120-200 ms). These observations support the fact that the hemodynamic fluctuations reported in this paper are a plausible physical explanation for the $S_{3}$ bruit.

Furthermore, if this sound is induced by the interaction of the flow instabilities and the LV tissue, it suggests that flow fluctuations are widespread in normal LV. Collins et al. ${ }^{11}$ showed on a large cohort that the $S_{3}$ bruit was detected in one third of asymptomatic individuals younger than 40 . Note that their technic was not able to detect quiet or intermittent $S_{3}$, underestimating potentially the already high prevalence of this sound.

Recently, Dyverfeldt et al. ${ }^{17}$ developed a new technique using CMR allowing the detection of velocity fluctuations and the estimation of their associated energy in vivo. Zajac et al. ${ }^{53}$ applied this technique on normal and myopathic LV and highlighted that the level of flow instabilities could be a relevant biomarker of abnormal flows in the ventricle. However, less intense fluctuations were also measured in normal ventricles. The fluctuations were observed in the basal third of the LV, with peaks associated with highest inflow velocity, i.e. peak $E$ and peak $A$ wave. For normal subjects, they reported a maximum of kinetic energy of $2.5+-1.2 \mathrm{~mJ}$ during diastole, which agree with the maximum value of $1.5 \mathrm{~mJ}$ obtained from our computation.

\subsection{Potential clinical implications}

As shown by Zajac et $a l^{53}$ a certain level of fluctuations intensity can be a sign of cardiac dysfunction. It is actually commonly accepted that flow fluctuations are synonym of pathologies in the cardiovascular system ${ }^{17,31}$. In light of the clinical observations discussed above and our results, it seems that non-negligible fluctuations in LV flows are actually not a synonym of 
pathological flow. Flow fluctuations in normal LV may be more common than thought so far, even though their intensity are an order of magnitude lower than the fluctuations found in the pathological cardiovascular flow previously reported ${ }^{16,18}$. In other words, the presence of flow fluctuations may reflect an altered LV function but should not be systematically considered as an absolute proof of a pathological state.

As CMR is widely used to explore the LV flow, the prevalence of fluctuations in normal LV serves as a reminder that time-resolved velocity fields measured with CMR are not instantaneous but mean flow fields ${ }^{15}$. The blood flow patterns ${ }^{49}$, blood residence times ${ }^{21}$ or any other quantity computed thanks to velocity gradients (pressure difference, vorticity, wall shear stress) correspond to the mean flow fields, not the actual in vivo flow. While the same mean flow as CMR is retrieved, Fig. 4 illustrates how different the instantaneous flow may be. The wall shear stress maps in Fig. 8 illustrate the consequences of these fluctuations on the cycle-to-cycle forces experienced by the heart cells.

Rather than the simple idea that fluctuations are a sign of pathology, we suggest that conclusions also depend on the levels of the fluctuations: flow fluctuations with significant energy content would indicate that substantial energy is transferred to fluctuations and thus results in a loss of energy. However, some fluctuations may be present, with small energy content, as in the present study: the energy loss due to "turbulence" is then small. In addition, to place the intensity and duration of the fluctuations in perspective, a rapid analysis shows that these fluctuations should not trigger blood damages. Consider first that the components of the fluctuations are similar in magnitude in all three directions, i.e. $u_{1}^{\prime} \approx u_{2}^{\prime} \approx u_{3}^{\prime} \approx$ $u^{\prime}$. This assumption allows writing FKE $\approx \frac{3 \rho}{2}<u^{\prime 2}>=\frac{3}{2} \sigma_{\text {Reynolds. }}$ The maximum FKE in the $\mathrm{LV}$ reached locally $150 \mathrm{~J} \mathrm{~m}^{-3}$, as described in the subsection 5.2 , which corresponds to an estimated maximum Reynolds stress component of $100 \mathrm{~J} \mathrm{~m}^{-3}$. This value is several times lower than the threshold values commonly cited for hemolysis ${ }^{30}$, especially for such a short exposure time. Some fluctuations may actually even be beneficial, especially during diastasis, to prevent the increase of effective viscosity due to aggregation at low shear rates ${ }^{7}$. These fluctuating velocities amplify the forces exerted by the blood on the LV wall cells, as shown by Fig 8 , and can potentially trigger mechanosensitive feedbacks. It has been shown in vitro that even weak flow stimuli can induce modifications of gene expression ${ }^{12,36}$ which in the LV, may result in its functional modification. Although the remodelling mechanisms of the LV are not completely understood and that the relevance of these fluctuating velocities compared with the mean flow field remains to be defined, fluctuations can be expected to play an important role in myocardial function and adaptations ${ }^{38}$. Such fluid dynamic considerations point toward interesting directions for future clinical research, complementing conventional studies focusing on the mean flow field ${ }^{39}$.

\subsection{Limitations}

An obvious limitation of our study is that we only simulate the LV of one subject under several modelling hypotheses and thus can only comment on the fluctuations detected in this 
simulation. Nevertheless, the results of this study are in accordance with several in vivo, in silico and in vitro evidence, as discussed above, and constitute a first step toward the study of these detected instabilities.

Some simplifications were also made. First, blood is here considered as Newtonian, as generally done in simulations of flows in large vessels. We did not account for the impact of the cordae tendinae of the MV or the trabeculations of the LV as they could not be captured by the CMR. These features could influence the dissipation of the instabilities ${ }^{51}$. The MV leaflet dynamics is not modelled: it may be speculated that their physiological flapping movement could enhance instabilities because of the added vortex shedding ${ }^{5}$ while the widening of the $\mathrm{MV}$ could decrease the flow rate coming in the LV, decreasing the generation of instabilities. The degree to which our MV model will modulate the turbulence characteristics can hardly be judged.

The medical images and the registration algorithm pilot the deformations of our imagebased LV. Thus, any images or registration flaws have an influence on the CFD results. A weak peak is visible on the MV flow rate at the very beginning of diastole: the quality of the CMR exam made it difficult for the registration algorithm to converge, generating indirectly this spurious peak. However, it can be speculated that this imperfection should have a limited impact on the presented results as the integrated FKE (Fig. 7) does only show a very weak inflexion corresponding to this event.

To be fully converged, the FKE statistics would need more cycles. However, a look at the computed FKE point-wise show that its cycle-averaged value using 20 or 30 cycles varies only by a few percent when the flow is the most disturbed. Figure S06 and Table S1 in the supplementary materials show it. Thus, while admittedly not fully converged, the statistics were judged sufficiently converged to conduct our study.

Finally, we also did not consider the impact of beat-to-beat variations of the heart rate as the LV deformation was extracted from CMR. However, taking into account for such variations would most probably enhanced the cycle-to-cycle fluctuations observed in this study where the boundary conditions and heart deformations are periodic in time.

\section{Acknowledgments}

The authors would like to express their gratitude to MD Dr. D. Coisne for many fruitful discussions. Dr. R. Moreno from the Rangueil University Hospital, Toulouse (France) is acknowledged for the CMR exams. Dr. V. Moureau and Dr. G. Lartigue from the CORIA lab, and the SUCCESS scientific group are acknowledged for providing the YALES2 code, which served as a basis for the development of YALES2BIO. This work was performed using HPC resources from GENCI-CINES (Grants 2014- and 2015-c2014037194). 


\section{References}

1. Barré, D., M. Kraushaar, G. Staffelbach, V. Moureau, and L. Y. M. Gicquel. Compressible and low Mach number LES of a swirl experimental burner. Comptes Rendus Mécanique 341:277287, 2013.

2. Baya Toda, H., O. Cabrit, K. Truffin, G. Bruneaux, and F. Nicoud. Assessment of subgrid-scale models with a large-eddy simulation-dedicated experimental database: The pulsatile impinging jet in turbulent cross-flow. Phys. Fluids 26:075108, 2014.

3. Carlsson, M., E. Heiberg, J. Toger, and H. Arheden. Quantification of left and right ventricular kinetic energy using four-dimensional intracardiac magnetic resonance imaging flow measurements. AJP Hear. Circ. Physiol. 302:H893-H900, 2012.

4. Celik, I. B., Z. N. Cehreli, and I. Yavuz. Index of Resolution Quality for Large Eddy Simulations. J. Fluids Eng. 127:949, 2005.

5. Charonko, J. J., R. Kumar, K. Stewart, W. C. Little, and P. P. Vlachos. Vortices formed on the mitral valve tips aid normal left ventricular filling. Ann. Biomed. Eng. 41:1049-1061, 2013.

6. Cheng, C. P., D. Parker, and C. A. Taylor. Quantification of Wall Shear Stress in Large Blood Vessels Using Lagrangian Interpolation Functions with Cine Phase-Contrast Magnetic Resonance Imaging. Ann. Biomed. Eng. 30:1020-1032, 2002.

7. Chien, S. Shear Dependence of Effective Cell Volume as a Determinant of Blood Viscosity. Science (80-. ). 168:977-979, 1970.

8. Chnafa, C. Using image-based large-eddy simulations to investigate the intracardiac flow and its turbulent nature. Montpellier: University of Montpellier, 2014, 231 pp.

9. Chnafa, C., S. Mendez, R. Moreno, and F. Nicoud. Using image-based CFD to investigate the intracardiac turbulence. In: Modeling the Heart and the Circulatory System, edited by A. Quarteroni. New-York: Springer International Publishing, 2015, pp. 97-117.

10. Chnafa, C., S. Mendez, and F. Nicoud. Image-based large-eddy simulation in a realistic left heart. Comput. Fluids 94:173-187, 2014.

11. Collins, S. P., P. Arand, C. J. Lindsell, W. F. Peacock, and A. B. Storrow. Prevalence of the Third and Fourth Heart Sound in Asymptomatic Adults. Congest. Hear. Fail. 11:242-247, 2005.

12. Davies, P. F., A. Remuzzi, E. J. Gordon, C. F. Dewey, and M. A. Gimbrone. Turbulent fluid shear stress induces vascular endothelial cell turnover in vitro. Proc. Natl. Acad. Sci. 83:2114-2117, 1986.

13. Domenichini, F., G. Pedrizzetti, and B. Baccani. Three-dimensional filling flow into a model left ventricle. J. Fluid Mech. 539:179, 2005.

14. Domenichini, F., G. Querzoli, a. Cenedese, and G. Pedrizzetti. Combined experimental and numerical analysis of the flow structure into the left ventricle. J. Biomech. 40:1988-1994, 2007.

15. Dyverfeldt, P., M. Bissell, A. J. Barker, A. F. Bolger, C.-J. Carlhäll, T. Ebbers, C. J. Francios, A. Frydrychowicz, J. Geiger, D. Giese, M. D. Hope, P. J. Kilner, S. Kozerke, S. Myerson, S. Neubauer, O. Wieben, and M. Markl. 4D flow cardiovascular magnetic resonance consensus statement. J. Cardiovasc. Magn. Reson. 17:72, 2015.

16. Dyverfeldt, P., M. D. Hope, E. E. Tseng, and D. Saloner. Magnetic resonance measurement of turbulent kinetic energy for the estimation of irreversible pressure loss in aortic stenosis. JACC. Cardiovasc. Imaging 6:64-71, 2013.

17. Dyverfeldt, P., J. P. E. Kvitting, A. Sigfridsson, J. Engvall, A. F. Bolger, and T. Ebbers. Assessment of fluctuating velocities in disturbed cardiovascular blood flow: In vivo feasibility of 
generalized phase-contrast MRI. J. Magn. Reson. Imaging 28:655-663, 2008.

18. Dyverfeldt, P., J.-P. E. Kvitting, C. J. Carlhäll, G. Boano, A. Sigfridsson, U. Hermansson, A. F. Bolger, J. Engvall, and T. Ebbers. Hemodynamic aspects of mitral regurgitation assessed by generalized phase-contrast MRI. J. Magn. Reson. Imaging 33:582-8, 2011.

19. Falahatpisheh, A., and A. Kheradvar. High-speed particle image velocimetry to assess cardiac fluid dynamics in vitro: From performance to validation. Eur. J. Mech. B/Fluids 35:2-8, 2012.

20. Glower, D. D., R. L. Murrah, C. O. Olsen, J. W. Davis, and J. S. Rankin. Mechanical correlates of the third heart sound. J. Am. Coll. Cardiol. 19:450-457, 1992.

21. Hendabadi, S., J. Bermejo, Y. Benito, R. Yotti, F. Fernández-Avilés, J. C. Del Álamo, and S. C. Shadden. Topology of blood transport in the human left ventricle by novel processing of doppler echocardiography. Ann. Biomed. Eng. 41:2603-2616, 2013.

22. Hult, P., T. Fjällbrant, B. Wranne, and P. Ask. Detection of the third heart sound using a tailored wavelet approach. Med. Biol. Eng. Comput. 42:253-258, 2004.

23. Kanski, M., P. M. Arvidsson, J. Töger, R. Borgquist, E. Heiberg, M. Carlsson, and H. Arheden. Left ventricular fluid kinetic energy time curves in heart failure from cardiovascular magnetic resonance 4D flow data. J. Cardiovasc. Magn. Reson. 17:111, 2015.

24. Khalafvand, S. S., E. Y. K. Ng, L. Zhong, and T. K. Hung. Fluid-dynamics modelling of the human left ventricle with dynamic mesh for normal and myocardial infarction: Preliminary study. Comput. Biol. Med. 42:863-870, 2012.

25. Kheradvar, A., and M. Gharib. On mitral valve dynamics and its connection to early diastolic flow. Ann. Biomed. Eng. 37:1-13, 2009.

26. Kilner, P. J., G. Z. Yang, a J. Wilkes, R. H. Mohiaddin, D. N. Firmin, and M. H. Yacoub. Asymmetric redirection of flow through the heart. Nature 404:759-761, 2000.

27. Kono, T., H. Rosman, M. Alam, P. D. Stein, H. N. Sabbah, D. Stein, and N. Wbbah. Hemodynamic correlates of the third heart sound during the evolution of chronic heart failure. Am. J. Med. 21:419-423, 1992.

28. Le, T. B., and F. Sotiropoulos. On the three-dimensional vortical structure of early diastolic flow in a patient-specific left ventricle. Eur. J. Mech. B/Fluids 35:20-24, 2012.

29. Long, Q., R. Merrifield, X. Y. Xu, P. Kilner, D. N. Firmin, and Y. G-Z. Subject-specific computational simulation of left ventricular flow based on magnetic resonance imaging. Proc. Inst. Mech. Eng. H. 222:475-485, 2008.

30. Lu, P. C., H. C. Lai, and J. S. Liu. A reevaluation and discussion on the threshold limit for hemolysis in a turbulent shear flow. J. Biomech. 34:1361-4, 2001.

31. Mann, D. L., D. P. Zipes, P. Libby, and R. O. Bonow. Braunwald's Heart Disease: A Textbook of Cardiovascular Medicine. Philadelphia: Elsevier Health Sciences, 2014, 2136 pp.

32. Markl, M., P. J. Kilner, and T. Ebbers. Comprehensive 4D velocity mapping of the heart and great vessels by cardiovascular magnetic resonance. J. Cardiovasc. Magn. Reson. 13:7, 2011.

33. Mendez, S., E. Gibaud, and F. Nicoud. An unstructured solver for simulations of deformable particles in flows at arbitrary Reynolds numbers. J. Comput. Phys. 256:465-483, 2014.

34. Mihalef, V., R. I. Ionasec, P. Sharma, B. Georgescu, I. Voigt, M. Suehling, and D. Comaniciu. Patient-specific modelling of whole heart anatomy, dynamics and haemodynamics from fourdimensional cardiac CT images. Interface Focus 1:286-296, 2011.

35. Nicoud, F., H. B. Toda, O. Cabrit, S. Bose, and J. Lee. Using singular values to build a subgridscale model for large eddy simulations. Phys. Fluids 23:1-35, 2011. 
current in vascular endothelial cells. Nature 331:168-70, 1988.

37. Pasipoularides, A. Diastolic filling vortex forces and cardiac adaptations: Probing the epigenetic nexus. Hell. J. Cardiol. 53:458-469, 2012.

38. Pasipoularides, A. Mechanotransduction mechanisms for intraventricular diastolic vortex forces and myocardial deformations: part 1. J. Cardiovasc. Transl. Res. 8:76-87, 2015.

39. Pedrizzetti, G., G. La Canna, O. Alfieri, and G. Tonti. The vortex--an early predictor of cardiovascular outcome? Nat. Rev. Cardiol. 11:545-53, 2014.

40. Pedrizzetti, G., and F. Domenichini. Left ventricular fluid mechanics: the long way from theoretical models to clinical applications. Ann. Biomed. Eng. 43:26-40, 2015.

41. Pedrizzetti, G., F. Domenichini, and G. Tonti. On the left ventricular vortex reversal after mitral valve replacement. Ann. Biomed. Eng. 38:769-773, 2010.

42. Pham, D. L., C. Xu, and J. L. Prince. Current methods in medical image segmentation. Annu. Rev. Biomed. Eng. 2:315-37, 2000.

43. Pope, S. B. Turbulent Flows. Cambridge: Cambridge University Press, 2000, 801 pp.doi:10.1088/0957-0233/12/11/705

44. Pope, S. B. Ten questions concerning the large-eddy simulation of turbulent flows. New J. Phys. 6:, 2004.

45. Querzoli, G., S. Fortini, and A. Cenedese. Effect of the prosthetic mitral valve on vortex dynamics and turbulence of the left ventricular flow. Phys. Fluids 22:1-10, 2010.

46. Sabbah, H. N., and P. D. Stein. Turbulent blood flow in humans: its primary role in the production of ejection murmurs. Circ. Res. 38:513-525, 1976.

47. Saber, N. R., N. B. Wood, a. D. Gosman, R. D. Merrifield, G. Z. Yang, C. L. Charrier, P. D. Gatehouse, and D. N. Firmin. Progress towards patient-specific computational flow modeling of the left heart via combination of magnetic resonance imaging with computational fluid dynamics. Ann. Biomed. Eng. 31:42-52, 2003.

48. Schenkel, T., M. Malve, M. Reik, M. Markl, B. Jung, and H. Oertel. MRI-Based CFD analysis of flow in a human left ventricle: Methodology and application to a healthy heart. Ann. Biomed. Eng. 37:503-515, 2009.

49. Töger, J., M. Kanski, M. Carlsson, S. J. Kovács, G. Söderlind, H. Arheden, and E. Heiberg. Vortex Ring Formation in the Left Ventricle of the Heart: Analysis by 4D Flow MRI and Lagrangian Coherent Structures. Ann. Biomed. Eng. 1-11, 2012.doi:10.1007/s10439-012-0615-3

50. Valen-Sendstad, K., and D. A. Steinman. Mind the gap: Impact of computational fluid dynamics solution strategy on prediction of intracranial aneurysm hemodynamics and rupture status indicators. Am. J. Neuroradiol. 35:536-543, 2014.

51. Vedula, V., J.-H. Seo, A. C. Lardo, and R. Mittal. Effect of trabeculae and papillary muscles on the hemodynamics of the left ventricle. Theor. Comput. Fluid Dyn. , 2015.doi:10.1007/s00162015-0349-6

52. Watanabe, H., S. Sugiura, and T. Hisada. The looped heart does not save energy by maintaining the momentum of blood flowing in the ventricle. Am. J. Physiol. Heart Circ. Physiol. 294:H2191-H2196, 2008.

53. Zajac, J., J. Eriksson, P. Dyverfeldt, A. F. Bolger, T. Ebbers, and C.-J. Carlhäll. Turbulent kinetic energy in normal and myopathic left ventricles. J. Magn. Reson. Imaging 41:1021-9, 2015. 